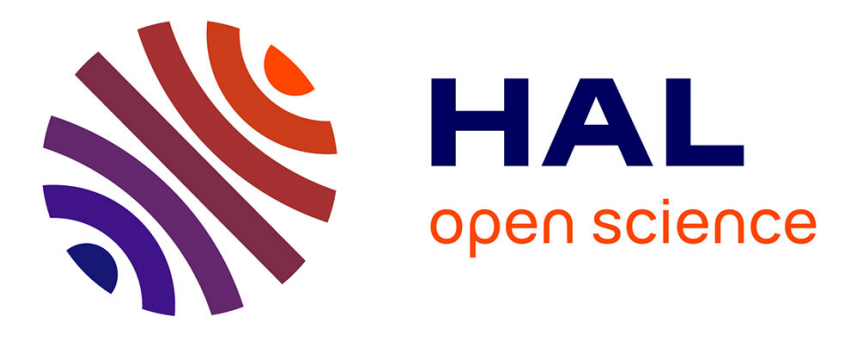

\title{
Patterning of a cohesionless granular layer under pure shear
}

\author{
Hector Alarcon, Jean-Christophe Géminard, Francisco Melo
}

\section{To cite this version:}

Hector Alarcon, Jean-Christophe Géminard, Francisco Melo. Patterning of a cohesionless granular layer under pure shear. Physical Review E : Statistical, Nonlinear, and Soft Matter Physics, In press. hal-01673512

\section{HAL Id: hal-01673512 https://hal.science/hal-01673512}

Submitted on 30 Dec 2017

HAL is a multi-disciplinary open access archive for the deposit and dissemination of scientific research documents, whether they are published or not. The documents may come from teaching and research institutions in France or abroad, or from public or private research centers.
L'archive ouverte pluridisciplinaire HAL, est destinée au dépôt et à la diffusion de documents scientifiques de niveau recherche, publiés ou non, émanant des établissements d'enseignement et de recherche français ou étrangers, des laboratoires publics ou privés. 


\title{
Patterning of a cohesionless granular layer under pure shear.
}

\author{
Héctor Alarcón ${ }^{1,2}$, Jean-Christophe Géminard ${ }^{3}$ and Francisco Melo ${ }^{1}$. \\ ${ }^{1}$ Departamento de Física, Universidad de Santiago de Chile, \\ Av. Ecuador 3493, Casilla 307, Correo 2, Santiago, Chile. \\ ${ }^{2}$ Departamento de Física, Facultad de Ciencias Fúsicas y Matemáticas, \\ Universidad de Chile, Casilla 487-3, Santiago, Chile. and \\ ${ }^{3}$ Université de Lyon, Laboratoire de Physique, Ecole Normale Supérieure de Lyon, \\ CNRS, UMR 5672, Laboratoire, ENS, Université, 46 Allée d'Italie, 69007 Lyon, France.
}

\begin{abstract}
The response of a thin layer of granular material to an external pure shear imposed at its base is investigated. The experiments show that, even for non-cohesive materials, the resulting deformation of the material is inhomogeneous. Indeed, a novel smooth pattern, consisting in a periodic modulation of the shear deformation of the free surface, is revealed by an image-correlation technique. These observations are in contrast with the observation of the fracture pattern previously observed in cohesive granular materials subjected to stretching. For cohesive materials, the instability is due to the weakening of the material which results from the rupture of capillary bridges that bond the grains to one another. For non-cohesive materials, the rupture of the capillary bridges cannot be invoked anymore. We show that the instability results from the decrease of friction upon shearing.
\end{abstract}

PACS: 89.75.Kd: Pattern formation in complex systems; 83.60.Uv: Rheology: fracture; 45.70.Qj: Pattern formation in granular matter

\section{INTRODUCTION}

Cohesive granular materials are characterized by a network of liquid bridges responsible of attractive capillary forces between particles [1]. Various regimes of cohesion are identified depending on the liquid content, leading to different scalings for the cohesion force [2, 4, 5]. Regardless of fluid content, a common feature of cohesive material is a weakening due to a decrease of both the associated adhesion force when a single bridge elongates [6] and the overall number of bonds which collapse when excessively stretched [7. This effect, which we referred to as strain softening [8], is observed above a critical stress associated with the cohesion due to the capillary bridges at grain contacts [9] and is responsible for the relatively low range of plasticity in cohesive granular materials.

In a recent work 8 we explored the tensile response of a horizontal layer of cohesive material subjected to homogeneous deformation in its bottom plane. We showed that "strain softening" is responsible for the nearly periodic modulation of the strain field along the pulling axis that develops as soon as the external deformation is imposed. The associated wavelength depends linearly on the layer thickness, is almost independent of particle size and depends linearly on the relative humidity. The flexural deformation of a cohesive granular layer reveals similar features [10]. To establish a more fundamental connection between the pattern features and the intrinsic properties of the granular material, we developed experimental methods for the assessment of the cohesion and shear modulus as function of the particles size and relative humidity [11.

Here, we explore the response of a granular layer to pure shear at its base. For important cohesion, we observe that cracks appear and form a periodic network. However, when cohesion is reduced, the layer deforms without fracturing and a pattern, consisting in rather a smooth, periodic, modulation of the layer thickness is observed, instead. For vanishing cohesion, the pattern does not disappear but the amplitude of modulation is so small that it is only revealed by the use of image correlation techniques. A simple model including, besides cohesion, the decrease of friction due to the induced dilation of the material accounts for the typical size of the structure.

\section{EXPERIMENTAL SETUP AND PROTOCOLS}

The experiment consists in imposing an in-plane deformation at the base of a thin layer of granular material. The mechanical system is composed of four linear actuators (Thorlabs Z825BV), placed in the $x-y$ axes (Fig. 1 ) (see Ref. 11 for details). Actuators are attached to four plexiglass blocks respectively, each of them holding one arm of a latex membrane cut in cross-shape (thickness $0.5 \mathrm{~mm}$, width $40 \mathrm{~cm}$ ). The membrane leans on a horizontal table that prevents bending due to weight and ensures planar deformation. Pure shear deformation at center is obtained by stretching two opposite arms and by shortening the arms in the perpendicular direction at the same velocity. We checked the amplitude and homogeneity of the deformation through digital image correlation.

The sample is prepared by pouring granular material into a circular mold of given height $h$ (from 1 to $10 \mathrm{~mm}$, to within $0.1 \mathrm{~mm}$ ) and internal diameter $7 \mathrm{~cm}$, leaning on the membrane, at center. The excess of grains is gently removed through the horizontal displacement a rod. After removal of the mold, we obtain a disk of granular material of well-defined thickness $h$. We used either glass or brass spherical-beads, the large density of brass making possible to explore the vanishing small cohesion 
regime, the weight of the grains overcoming more easily the capillary forces. Two ranges of particle diameter $d$ are considered for both materials: $(0-45)$ or $(150-200) \mu \mathrm{m}$ for glass, and $(75-106)$ or $(212-300) \mu \mathrm{m}$ for brass.

The whole experimental device is placed in a chamber whose atmosphere is maintained at constant relative humidity, $R_{H}$ [equilibrated with saturated salt solutions and monitored using a humidity meter (Lutron HT-3015)]. Prior to any experimental run, particles are sonicated in acetone, rinsed in pure water and dried, to prevent undesired cohesion due to contamination. Cohesion is measured, in addition, as described in Ref. [11].

The free surface of the sample is imaged from above by using a digital camera (Nikon DMX1200). An annular light-source (ring of LEDs, Fig. 1) placed $1 \mathrm{~cm}$ above the sample, at center, provides the grazing lighting adequate to observation of tiny displacements at the layer surface.

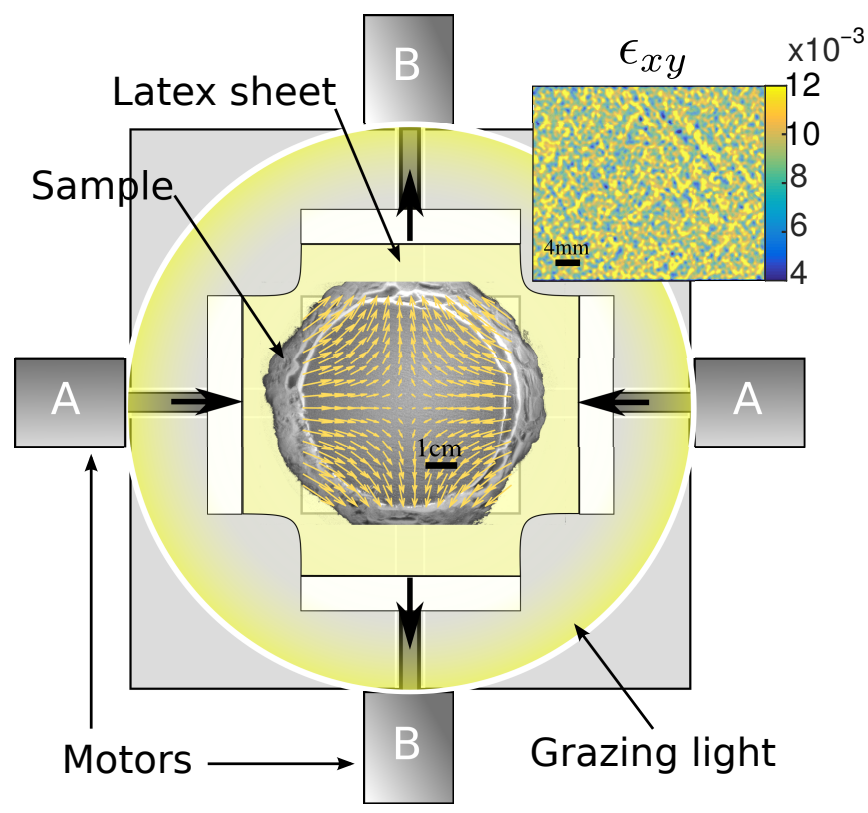

FIG. 1. (Color online)-Sketch of the experimental setup The 4 arms of a latex membrane are driven by computercontrolled actuators. At center, arrows show the displacements of the membrane. Pure shear strain is achieved to better than $1 \%$ over a surface area of $50 \mathrm{~cm}^{2}$. Inset: typical modulation of shear strain at the free surface [Brass beads, $d=(75-106) \mu \mathrm{m}, h=3 \mathrm{~mm}, \theta=0.14$ and $\left.R_{H}=39 \%\right]$.

The typical experimental run consists in applying the pure-shear deformation at the membrane in quasi-static manner and in determining precisely the displacement fields at the free surface of the granular layer. In practice, the imposed shear strain, $\theta$ is increased by constant steps $\Delta \theta$, of the order of $10^{-3}$. After each step, the free surface at rest is imaged with a resolution of $(3840 \times 3072) \mathrm{px}^{2}$. In order to extract the resulting displacement field, the following correlation procedure is then applied to the successive images: We define a sliding window (typical surface area $1.5 \mathrm{~mm}^{2}$, containing enough particles to be considered as a coarse grain), and scan the whole image (typical displacement $0.2 \mathrm{~mm}$ ). The procedure achieves a spatial resolution of the order of $1 \mathrm{~mm}$ and a resolution of the order of a few $\mu \mathrm{m}$ in the local grain displacement. Shear and vertical vorticity fields are obtained through differentiation of displacement field [11.

\section{EXPERIMENTAL RESULTS}

When the thickness, $h$, and the cohesion, $\sigma_{s}$, are sufficiently large, we observe fracturing of the granular layer (Fig. 2). The fractures organize in rather periodic pattern. The typical distance between neighbour fractures, $\lambda$, increases with both the layer thickness and cohesion.
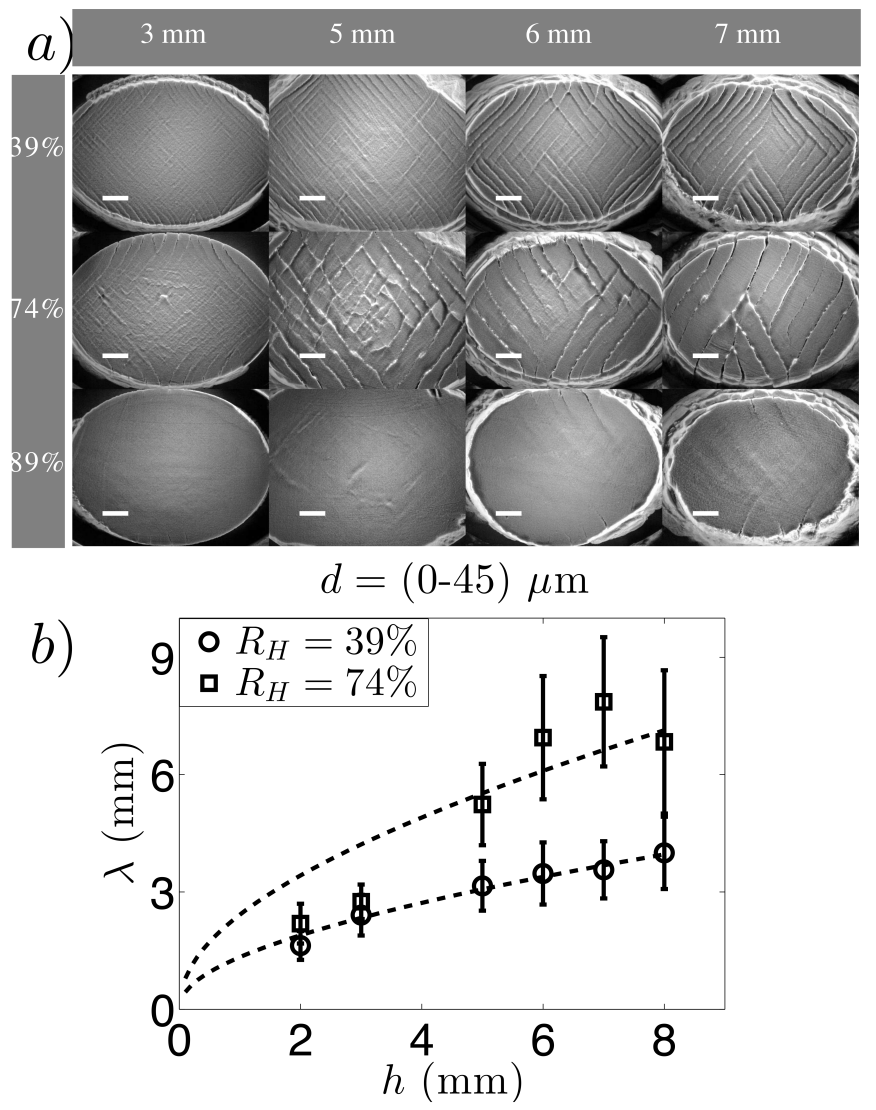

FIG. 2. (Color online) a) Pattern structure for various $h$ and humidity. $R_{H}\left(\sigma_{s}\right): 39 \%(17 \mathrm{~Pa}), 74 \%(37 \mathrm{~Pa}), 89 \%$ $(144 \mathrm{~Pa})$, scale bar $=1 \mathrm{~cm}$. b) Pattern wavelength, $\lambda$, for increasing $h$ at various cohesion (relative humidity, $R_{H}$ ). The lines are from Eq. (4) with parameters reported in Table I [Glass beads, $d=(0-45) \mu \mathrm{m}, \Delta \theta=1.5 \times 10^{-3}$ ].

It is of particular interest that a pattern is still present even when fracturing is not observed in Fig. 2 ( $h=3 \mathrm{~mm}$ and $R_{H}=39 \%$, for instance). This first observation is confirmed by the results obtained with larger grains (Fig. 3): even if not visible on simple images of the free surface, the squared patterns is revealed by the shear 
or vorticity fields, whatever the thickness and relative humidity in the experimental range (Fig. 4). In addition, note that, as expected, the typical distance between neighboring fractures is no longer a function of $R_{H}$ in the limit of vanishing cohesion (Fig. $3 \mathrm{p}$ ).

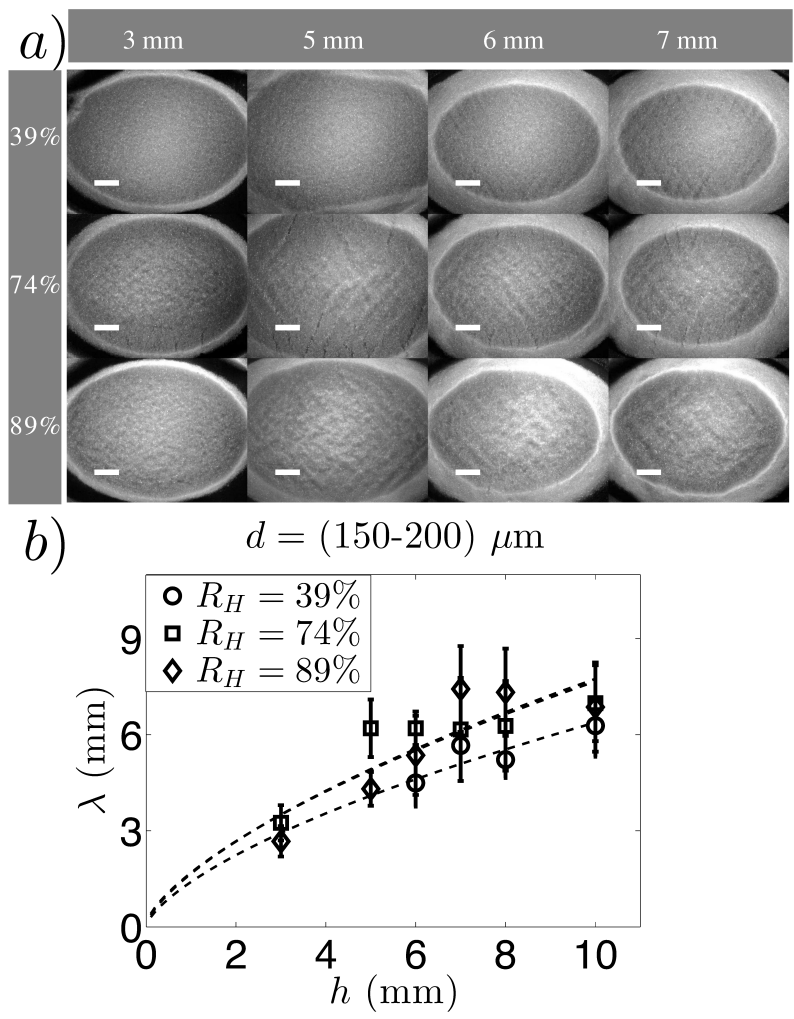

FIG. 3. (Color online) a) Pattern structure for various $h$ and humidity $R_{H}\left(\sigma_{s}\right): 39 \%(0.5 \mathrm{~Pa}), 74 \%(0.8 \mathrm{~Pa}), 89 \%(1.9 \mathrm{~Pa})$, scale bar $=1 \mathrm{~cm}$. b) Wavelength $\lambda$ for increasing $h$ at various cohesion. The lines are from Eq. (4) with parameters reported in Table I] [Glass beads, diameter (150-200) $\mu \mathrm{m}, \Delta \theta=1.5 \times$ $\left.10^{-3}\right]$

The previous results reveal that, upon shear, the granular layer is subjected to an instability leading to a modulation of the in-plane strain field. A sensitive method to determine the onset of instability consists in considering the amplitude of the modulation of the grain displacement at the free surface as function of the shear-strain imposed in the bottom plane. In order to measure a representative averaged amplitude of the modulation, we select a line profile along a perpendicular to the fractures. The displacement along the fracture, $\Delta d$, is then measured revealing a well-defined modulation at the pattern wavelength, $\lambda$. In Fig. 5, we report the averaged amplitude of such modulation, $\langle\Delta d\rangle$, as a function of $\theta$. We observe that, $\langle\Delta d\rangle$ is nearly zero for small $\theta$, but significantly increases for $\theta$ above a critical value. In addition, we note that the maximum of the averaged amplitude $\langle\Delta d\rangle$ increases with the layer thickness, $h$. Even if the behavior of the onset as function of $h$ is difficult to assess from our experimental data sets, the onset

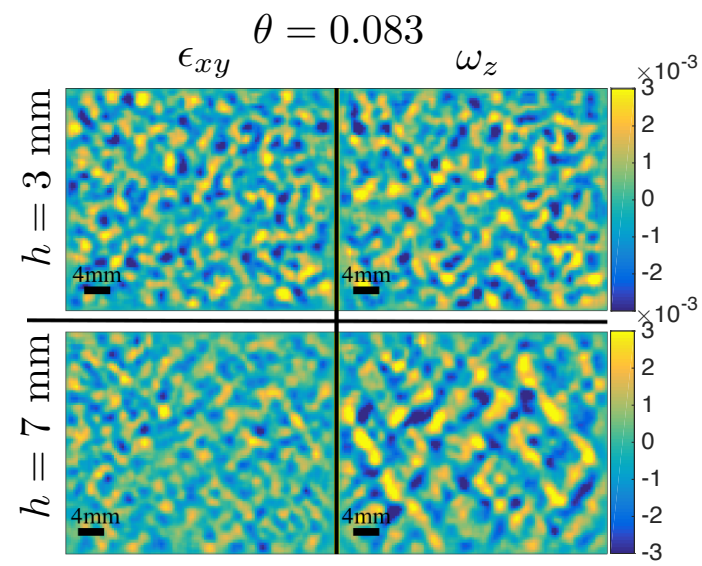

FIG. 4. (Color online) Surface shear, $\epsilon_{x y}$, and vorticity, $\omega_{z}$, fields for two distinct values of thickness, for $\theta=0.083$ [Glass beads, diameter (150-200) $\left.\mu \mathrm{m}, \Delta \theta=1.5 \times 10^{-3}, R_{H}=39 \%\right]$.

of instability is clearly smaller for larger cohesion, at a given $h$.

In order to explore the regime of vanishing cohesion in the same range of grain size, we now use brass particles and reduce the relative humidity as much as possible. In a dry system, the cohesion originates from the Van der Waals attraction force between the particles. We estimate from the Hamaker's constant, $A_{H} \approx 4 \times 10^{-19} \mathrm{~J}$, and from the typical size of the asperities at the particles surface, $D \approx 200 \mathrm{~nm}$ (obtained with an atomic force microscope [11), the tensile stress associated with an individual asperity, separated from a flat surface by a distance $z$ of atomic scale $\left(z=z_{a} \approx 1 \mathrm{~nm}\right)$ is about $A_{H} D /\left(3 d^{2} z_{a}^{2}\right) \approx 0.02 \mathrm{~Pa}$. Considering that cohesion is due to the contribution of all asperities, of surface density $\rho_{s}$, in the region of contact, assuming the separation $z=z_{a}+2 r^{2} / d$ at a distance $r$ from the center of the contact region, we get $\sigma_{s} \approx \pi A_{H} D \rho_{s} /\left(3 d z_{a}\right)$. From [11, we estimate that the distance between asperities is of about $5 D$, such that $\rho_{s} \approx 1 /(5 D)^{2}$ and, thus, $\sigma_{s} \approx 1 \mathrm{~Pa}$. This value is much smaller that the typical pressure $P=\rho g h \approx 400 \mathrm{~Pa}$ (with $\rho_{b}=8 \times 10^{3} \mathrm{~kg} / \mathrm{m}^{3}$ and $h \approx 0.5 \mathrm{~cm}$ ) at the base of the granular layer. When shear is imposed to a such non-cohesive granular material, the cellular pattern is revealed by image correlation analysis, only. The vorticity fields are the most suitable to reveal the structure (Fig. 6). We again observe that the wavelength, $\lambda$, increases with the layer thickness, $h$. We also note that $\lambda$ increases with the grain size.

\section{ANALYSIS}

Our analysis of the system behavior is based on the simplest heuristic model accounting for friction and, eventually, cohesion.

When a dry granular material is deformed, the main energetic cost is from the solid friction between the grains 

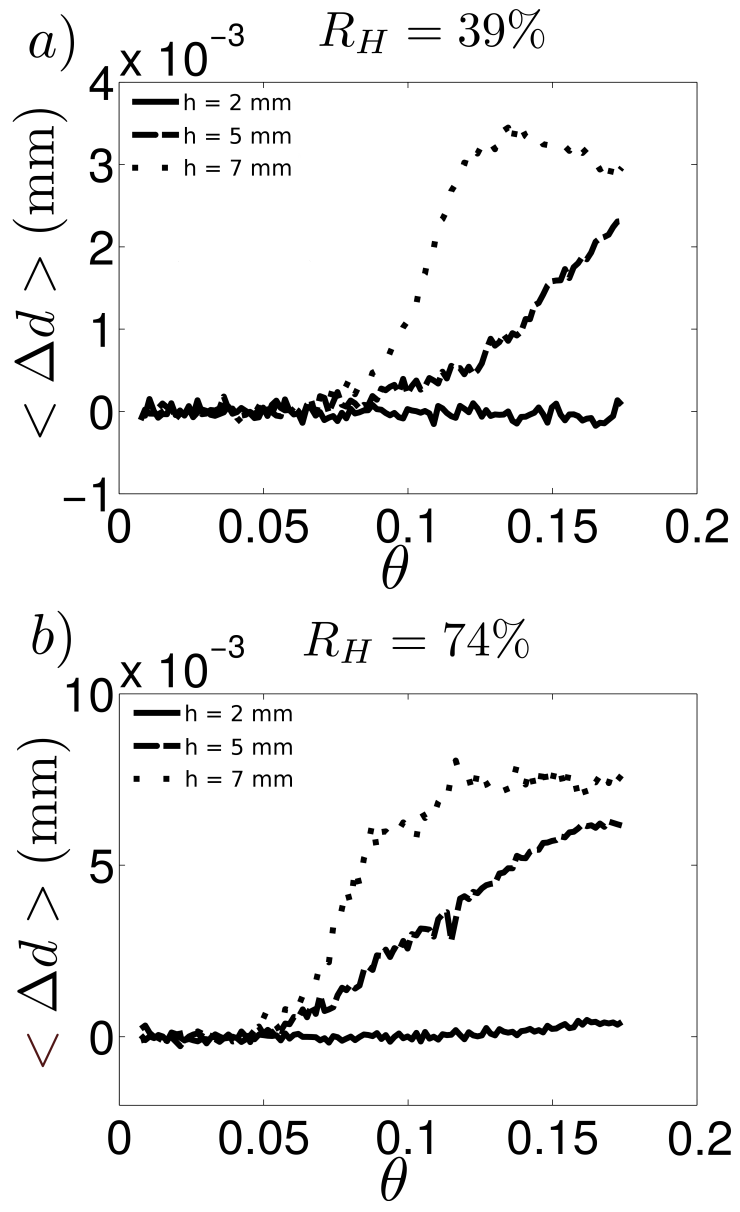

FIG. 5. (Color online)-Amplitude $\langle\Delta d\rangle$ of the modulation vs. shear-strain $\theta-$ a) $R_{H}=39 \%$ and b) $R_{H}=74 \%$ [Glass beads, diameter $\left.(0-45) \mu \mathrm{m}, \Delta \theta=1.5 \times 10^{-3}\right]$.

[8, 10, 11. The simplest way to account for friction is to write that, when two surfaces in regard are displaced with respect to one another, the energetic cost is proportional to the friction coefficient $\mu$ and local pressure $P$. However, the friction is known to decrease upon shear due to the dilation of the material, an assumption that is well supported by early works 12 . Starting from the initial value $\mu_{s}$ previous to shear, the friction coefficient is decreased by $\Delta \mu$ for a typical displacement $\delta$ (of the order of the grain radius) of the two surfaces in regard. Then, the friction coefficient remains constant and equal to $\mu_{d} \equiv \mu_{s}-\Delta \mu$. Here, seeking for simplicity, we will assume that the frictional coefficient is constant and equal to $\mu=\mu_{d}$ once the material is sufficiently deformed, but that an additional energetic cost, $\Gamma=\Delta \mu P \delta$, is to be paid to initially deform the material.

In a dry granular material, the local pressure $P$ is due to the weight of the grains, only. We have, at a distance $z$ from the free surface, $P=\rho g z$. In order to account for the results obtained for small, but not negligible cohesion, we generalize slightly the model by introducing
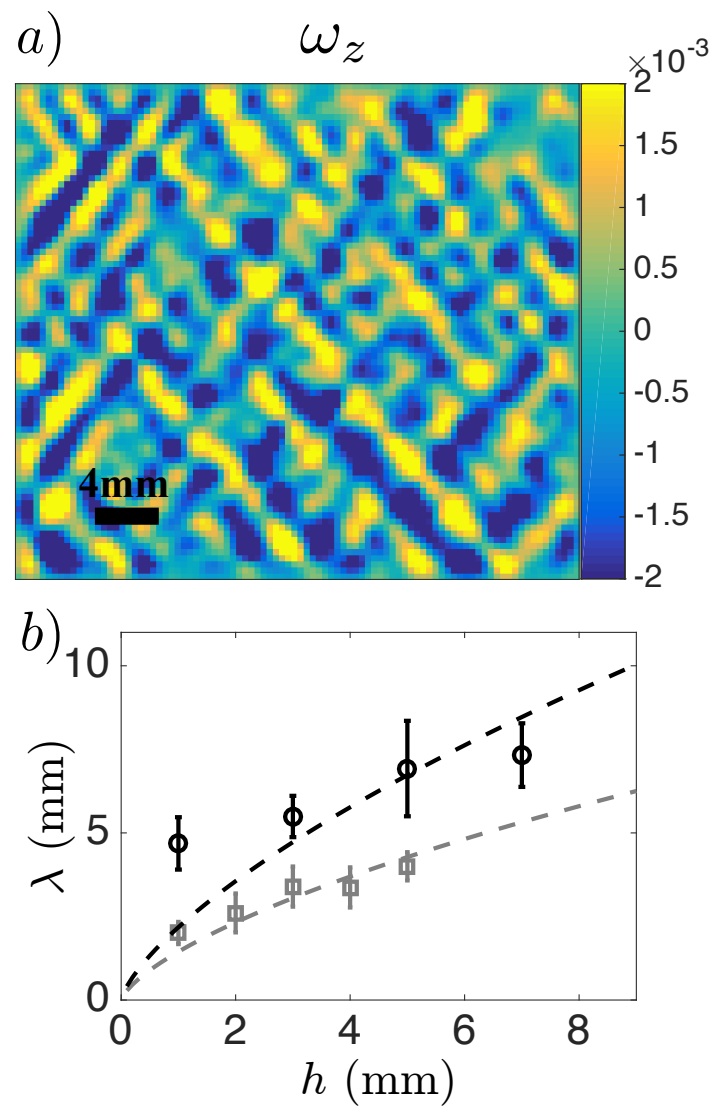

FIG. 6. (Color online)-Vorticity fields $\omega_{z}$ for brass particles a) $d=(75-106) \mu \mathrm{m}\left[h=5 \mathrm{~mm}, \theta=0.14\right.$ and $\left.R_{H}=39 \%\right]$. b) Wavelength $\lambda$ vs. thickness $h,(\square): d=(75-106) \mu \mathrm{m}$ and (o) : $d=(212-300) \mu \mathrm{m}$. The lines are from Eq. (4) with parameters reported in Table II] [Brass beads, $\Delta \theta=$ $3.6 \times 10^{-3}, \theta=0.14$ and $R_{H}=39 \%$.

the energetic cost associated with the liquid bridges. To do so, we take into account that the liquid bridges are responsible for a tensile pressure $\sigma_{s}$, independent of the depth $z$, which must be added to the pressure $P$. However, when the material is deformed, $\sigma_{s}$ works only on a distance $d_{b}$ that is of the order of the bridge size. For the humidity content considered here, the typical size of the capillary bridge is of the order of the size of the asperities at the grain surface. Thus, $d_{b} \ll \delta$. When the relative displacement of two grains exceeds $\delta$, the bridges are broken. Considering that the force associated with the tensile pressure only works over the distance $d_{b}$, we obtain that the contribution of the cohesion to $\Gamma$ is of the order of $(\mu+\Delta \mu) \sigma_{s} d_{b}$. In the following, we thus consider that $\Gamma=\Delta \mu P \delta+\mu \sigma_{s} d_{b}$, where we considered $\Delta \mu \ll \mu$.

Let us assume that, upon uniform shear in the bottom plane, the granular layer forms a series stripes of width $\lambda$ that are not deformed, but separated from the membrane and from one another by shear bands. The energetic cost associated with the deformation is thus the sum of the cost of the shear band in the bottom plane, plus the 


\begin{tabular}{|l|c|c|c|c|c|c|}
\hline Glass & \multicolumn{3}{|c|}{$(0-45) \mu \mathrm{m}$} & \multicolumn{3}{c|}{$(150-200) \mu \mathrm{m}$} \\
\hline \hline$\sigma_{s}(\mathrm{~Pa})$ & 17 & 37 & 144 & 0.5 & 0.8 & 1.9 \\
\hline$\sigma_{s} / \rho g h_{0}$ & 0.17 & 0.37 & 1.44 & 0.005 & 0.008 & 0.019 \\
\hline$\frac{\alpha \delta \Delta \mu}{\mu^{2}}(\mathrm{~mm})$ & 2.85 & 5.0 & - & 8.3 & 14.1 & 13.6 \\
\hline
\end{tabular}

TABLE I. Parameter $\alpha \delta \Delta \mu / \mu^{2}$ vs. $\sigma_{s}$ for glass particles.

cost of the vertical shear bands. At bottom, we have: $E_{h}=\Gamma_{h} \lambda+\mu \rho g h \int_{-\lambda / 2}^{+\lambda / 2} \theta x d x$. The first contribution $\Gamma_{h} \lambda$ is the cost of the fracture at the bottom which is the sum of the excess energy until the liquid bridges are broken, $\mu \sigma_{s} d_{b}$, and of the excess energy to initiate the shear deformation, $\Delta \mu \rho g h \delta$. The second contribution is that of friction, the local pressure being $P=\rho g h$. Considering the dimensionless cost per unit length $\mathcal{E}_{h} \equiv$ $E_{h} /(\mu \rho g h \lambda)$, we write:

$$
\mathcal{E}_{h}=\epsilon d_{b}+\frac{\Delta \mu}{\mu} \delta+\frac{\theta}{4} \lambda
$$

where we introduced $\epsilon \equiv \sigma_{s} /(\rho g h)$. In the same way, at the vertical walls between the bands, we have: $E_{v}=$ $\Gamma_{v} h+\mu \rho g\left[\int_{0}^{h}(h-z) d z\right] \theta \lambda$ where the cost of the vertical wall is $\Gamma_{v}=\mu \sigma_{s} d_{b} h+\Delta \mu \rho g(h / 2) \delta h$ (the pressure $P$ is averaged over the thickness). The dimensionless cost per unit length is:

$$
\mathcal{E}_{v}=\frac{1}{2}\left(2 \epsilon \frac{d_{b}}{\lambda}+\frac{\Delta \mu}{\mu} \frac{\delta}{\lambda}+\theta\right) h .
$$

The sum $\mathcal{E}_{h}+\mathcal{E}_{v}$ is the total cost (i.e. the energy lost by friction) per unit length in the volume upon deformation of the granular layer.

In order to determine if the layer is unstable (with the respect of the modulation with the wavelength $\lambda$ ) and to obtain the most unstable wavelength, we first estimate the energy $\mathcal{E}_{s}$ that would be elastically loaded in the system, supposed to deform homogeneously, for the same imposed shear at the bottom. The energy of this homogeneous state is obtained, provided the knowledge of the shear modulus $G$ of the granular layer, by writing $E_{s}=\lambda \int_{0}^{h} \frac{1}{2} G \theta^{2} d z$. In the absence of cohesion, $G=\alpha P$ where $\alpha=E d /\left(6 J l_{R}\right)$ [11. The prefactor $\alpha$ thus depends on physical properties of the grains, such as the Young modulus $E$, the yield stress $J$ and the typical size of the surface asperities $l_{R}$. We have:

$$
\mathcal{E}_{s}=\frac{1}{4} \frac{\alpha}{\mu}(1+2 \epsilon) h \theta^{2},
$$

where the coefficient $\mu$ appears only because our choice of the energy scale (i.e. $\mu \rho g h \lambda$ ).

In order to determine the onset of the instability and the most unstable wavelength, we compare the total loss by friction, $\mathcal{E}_{h}+\mathcal{E}_{v}$, and $\mathcal{E}_{s}$. We assume that the energy that would be loaded elastically in absence of modulation, $\mathcal{E}_{s}$, is entirely dissipated by friction, which is somehow equivalent to the application of a principle of maximum energy release rate commonly used in fracture theory [13]. We thus write $\mathcal{E}_{h}+\mathcal{E}_{v}=\mathcal{E}_{s}$, which is reasonable for a stiff system $(G \gg \mu P)$. We thus have a first relation between $\theta$ and $\lambda$. Then, we obtain the onset from the value of $\lambda$ that minimizes $\theta$. We get that, at the onset, $\theta \lambda^{2}=2 h\left[2 \epsilon d_{b}+\delta \Delta \mu / \mu\right]$ together with:

$$
\left(\frac{\lambda}{h}\right)^{4}+\left(\frac{\lambda}{h}\right)^{3}+\left(\frac{\lambda}{h}\right)^{2}=\frac{\alpha}{\mu} \frac{\Delta \mu}{\mu} \frac{\delta}{h}\left(1+2 \frac{\sigma_{s}}{\rho g h}\right) .
$$

where we took into account that $\epsilon d_{b} / \delta \ll 1$.

In order to contrast predictions from our theoretical arguments with the experimental results, we adjust the wavelength measured with glass beads for several cohesions using $\alpha \delta \Delta \mu / \mu^{2}$ as single fitting parameter (Figs. 2 and 3 and report the results in Table I The importance of cohesion expressed trough the ratio $\sigma_{s} / \rho g h_{0}$ is shown in Table I] where the typical thickness of the layer is $h_{0}=5 \mathrm{~mm}$. The above analysis, along with the obtained values of $\sigma_{s} / \rho g h_{0} \approx 1$, indicates that the characteristics of the structures observed with small particles are strongly influenced by the cohesion (Fig. 2). On the contrary, for larger particles, $\sigma_{s} /\left(\rho g h_{0}\right) \ll 1$, and null influence of the cohesion is expected in this limit. According to Eq. (4), all data presented in Fig. 3 collapse together within the errors bars. Finally, we report in Table II a summary of all the experimental parameters. The typical size $l_{R}$ of the surface asperities for glass and brass particles are obtained by atomic force measurements as discussed in [11. The values of the $E / J$ for glass and brass are from the literature. We assume a value of frictional coefficient $\mu=0.5$ and $\Delta \mu=0.1$ in all cases and estimate the characteristic length $\delta$. We observe that $\delta$ is of the order of a few micrometers, thus a fraction of the grain size. Even if no clear dependence of $\delta$ on the experimental parameters can be deduced from our measurements, the order of magnitude is in agreement with the physical meaning of this parameter, i.e. the relative displacement of two granular surfaces with respect to one another for the system to dilate, and thus to reach the constant value $\mu$ of the friction coefficient. Moreover, we note that such distance $\delta$ is compatible with a typical shear deformation $\theta$ at the onset of the order of $\delta / d$, thus of a few percents as observed experimentally.

\begin{tabular}{|l|c|c|c|c|}
\hline & \multicolumn{2}{|c|}{ Glass } & \multicolumn{2}{c|}{ Brass } \\
\hline$d(\mu \mathrm{m})$ & $0-45$ & $150-200$ & $75-106$ & $212-300$ \\
\hline$l_{R}(\mu \mathrm{m})$ & 0.1 & 0.07 & 1 & 1 \\
\hline$E / J$ & $25-40$ & $25-40$ & $250-300$ & $250-300$ \\
\hline$\alpha$ & 1700 & 7500 & 3000 & 8500 \\
\hline \hline$\delta(\mu \mathrm{m})$ & $\sim 5$ & $\sim 4.4$ & $\sim 8$ & $\sim 11$ \\
\hline
\end{tabular}

TABLE II. Summary of the experimental parameters. 


\section{CONCLUSIONS}

In conclusion, we have evidenced that sheardeformation of a granular material strongly depends on the cohesion. For $\sigma_{s} / P \gtrsim 1$, the material breaks in a series of parallel cracks. In contrast, for $\sigma_{s} / P \ll 1$, the granular layer deforms without fracturing, but a square pattern consisting in a smooth, periodic, modulation of the layer thickness is observed. At low cohesion the weakening of the liquid bridges, which is at play at large cohesion, cannot be invoked anymore. We show that the decrease of the friction, associated with the shear-induced dilation of the material, can explain the instability observed in dry materials. Our model recovers the correct dependence of the characteristic length of the pattern on the layer thickness.

The authors acknowledge the support from LIA-MSD, CNRS-France, and Conicyt-Chile through the program Fondecyt Anillo ACT-1410. F.M. acknowledges "Convenio de Desempeño" $\mathrm{N}^{0} 1555$ UdeSantiago and the invitation as professor by the École Normale Supérieure de Lyon (ENS-Lyon) and H. A. the support of Fondecyt postdoctoral program, under grant 3160341.
[1] Z. Fournier, et. al., J. Phys.: Condens. Matter 17, S477S502 (2005).

[2] M. Scheel, R. Seemann, M. Brinkmann, M. Di Michiel, A. Sheppard, B. Breidenbach and S. Herminghaus, Nature 7, 189-193 (2008).

[3] L. Bocquet, E. Charlaix, S. Ciliberto and J. Crassous, Nature 396 735-737 (1998).

[4] S. Nowak, A. Samadani and A. Kudrolli, Nature Physics, 1, 50-52 (2005).

[5] T. C. Halsey and A. J. Levine, Phys. Rev. Lett. 80, 31413144 (1998).

[6] J. Crassous, E. Charlaix, and J.L. Loubet, Phys. Rev. Lett. 78, 2425-2428 (1997).

[7] C. D. Willett, M. J. Adams, S. A. Johnson and J. P. K.
Seville, Langmuir 16, 9396-9405 (2000).

[8] H. Alarcón, O. Ramos, L. Vanel, F. Vittoz, F. Melo and J.-C. Géminard Phys. Rev. Lett. 105, 208001 (2010).

[9] T. Gröger, U. Tüzün and D. M. Heyes, Powder Tech. 133, 203-215 (2003).

[10] J.-C. Géminard, L. Champougny, P. Lidon and F. Melo, Phys. Rev. E. 85, 012301 (2012).

[11] H. Alarcón, J.-C. Géminard and F. Melo Phys. Rev. E. 86 (6), 061303 (2012).

[12] F. Tapia, D. Espíndola, E. Hamm, F. Melo, Phys. Rev. E 87, 014201 (2013).

[13] F. Francfort and J.-J. Marigo, J. Mech. Phys. Solids 46, 1319 (1998). 